\title{
Stage 0 Penile Cancer AJCC v6
}

National Cancer Institute

\section{Source}

National Cancer Institute. Stage O Penile Cancer A/CC v6. NCI Thesaurus. Code C3643.

Stage 0 includes: ( $\mathrm{T}$ is, NO, MO) and (Ta, NO, MO). T is: Carcinoma in situ. Ta: Non-invasive verrucous carcinoma. N0: No regional lymph node metastasis. M0: No distant

metastasis. (AJCC 6th ed.) - 2003 